\title{
Towards a Novel Vaccine against Human Cytomegalovirus Based on a Chimeric Ad5F35 Adenovirus Vector Expressing the Immunodominant Antigenic Domain 1 Epitope
}

\author{
Ping Zhao a,b Daoxin Ma ${ }^{a}$ Shuxin Yan ${ }^{a} \quad \mathrm{NaShao}^{\mathrm{a}} \quad$ Jingru Zhang ${ }^{\mathrm{a}}$ Zheng $\mathrm{Bi}^{\mathrm{a}}$ \\ Jianjian Dai ${ }^{a} \quad$ Min Ji $^{a}$ Chunyan $\mathrm{Ji}^{\mathrm{a}}$ \\ a Department of Hematology, Qilu Hospital, Shandong University, and ${ }^{b}$ Department of Pediatrics, \\ Jinan Central Hospital Affiliated to Shandong University, Jinan, PR China
}

\section{Key Words}

Cytomegalovirus, human - Vaccine $\cdot$ Antigenic domain-1 Adenoviral vector, Ad5F35 • Peripheral blood mononuclear cells

\begin{abstract}
Background: Antibodies induced from glycoprotein B (gB) by antigenic domain (AD)-1 demonstrate broad neutralizing activity across different human cytomegalovirus (HCMV) types. This study aimed to prepare a novel HCMV vaccine using the modified adenoviral vector Ad5F35 to direct the expression of the conserved HCMV epitope AD-1 and to determine its transfer and expression in peripheral blood mononuclear cells (PBMCs). Methods: AD-1 genes were amplified from AD169 HCMV strain and cloned into the Ad5F35 vector. Ad5F35-AD-1 virus vaccine was prepared by packaging Ad5F35-AD-1 into HEK293 cells. RT-PCR and fluorescence detection were used to detect the expression of $A D-1$ in HEK293 cells. PBMCs were stimulated in vitro with Ad5F35$A D-1$ virus vaccine. The AD-1 expression in PBMCs was determined with immunocytochemistry and cell viability was measured to observe the possible adverse effects of AD-1 on PBMCs. Results: We constructed an Ad5F35-AD-1 vector and transferred it into HEK293 cells to prepare the Ad5F35-AD-1 virus vaccine successfully. The AD-1 gene was proved to be
\end{abstract}

expressed in HEK293 cells. In vitro stimulation of PBMCs with Ad5F35-AD-1 showed the highly efficient expression of AD-1 and low cytopathic activity in PBMCs. Conclusion: The novel vaccine Ad5F35-AD-1 is a promising candidate for clinical trials and may be of utility in prime-boost strategies for HCMV prevention and control.

Copyright $\odot 2009$ S. Karger AG, Basel

\section{Introduction}

Human cytomegalovirus (HCMV) is a member of the herpesvirus family. The virus is a major cause of morbidity and mortality in congenitally infected infants and in recipients of hematopoietic stem cells and solid organ transplants [1-3]. Although antiviral drugs have reduced the risk of HCMV disease in transplant recipients, these are associated with potentially serious side effects and do not prevent late-onset HCMV disease [4]. They are also of only limited utility in congenital HCMV disease. An effective HCMV vaccine to prevent or reduce HCMV-associated disease is a high priority.

Early studies indicated that HCMV glycoprotein B (gB) is the major target of the neutralizing antibodies induced by naturally acquired HCMV infection [5]. Accordingly, many attempts have been made to develop a

\section{KARGER}

Fax +41 613061234 E-Mail karger@karger.ch www.karger.com
(C) 2009 S. Karger AG, Basel

0300-5526/09/0521-0035\$26.00/0

Accessible online at:

www.karger.com/int
Daoxin $\mathrm{Ma}, \mathrm{PhD}, \mathrm{MD}$

Department of Hematology, Qilu Hospital

Shandong University, 107 WenhuaXi Road

Jinan 250012 (PR China)

Tel. +86 5318216 9887, Fax +865318692 7544, E-Mail daoxinma@hotmail.com 
subunit vaccine based on $\mathrm{gB}$. Vaccines expressing recombinant $g B$ protein from plasmid DNA or from recombinant vaccinia (modified virus Ankara) have been investigated in animal models [6-8]. Safety and moderate immunogenicity have been demonstrated with these vaccines, but no licensed HCMV vaccine has yet been made available.

Epitopes in gB, particularly the antigenic domain-1 (AD-1), have proved to be important for the induction of virus-neutralizing humoral immunity. Clonal diversification due to somatic mutation is thought to account for the broad spectrum of antibody antiviral activity generated following immunization [9]. Furthermore, a plasmid expressing truncated and secreted forms of $\mathrm{gB}$ induced higher levels of HCMV-neutralizing antibody in mice and was more immunogenic than plasmids expressing full-length $\mathrm{gB}[8,10]$. These findings have implications for the development of a subunit vaccine based on AD-1.

The modified adenoviral vector Ad5F35, which is a fiber-substituted Ad5 vector containing Ad35 fiber proteins, possesses more expanded tropism and larger foreign DNA package ability than the conventional Ad5 vectors without affecting the viral growth rate and titer [11]. It exhibits a high transduction efficiency in human hematopoietic progenitors, dendritic cells, and mesenchymal stem cells with the minimum cytotoxic effect $[12$, 13]. It is able to transduce cells via cellular receptor human CD46 [14]. The vector has a high safety margin due to its inability to replicate in human cells, and has been approved for human testing by the US Food and Drug Administration. The safety and efficacy of Ad5F35 make it an ideal live viral vector for the construction of an HCMV vaccine. We have used this expression system to direct the expression of the highly conserved conformational epitope AD-1 from gB protein of HCMV. We report that the adenovirus-based vaccine replicates to high titer, efficiently infects human peripheral blood mononuclear cells (PBMCs), and can induce a potent lymphoproliferative response.

\section{Materials and Methods}

\section{Culture of Cells and HCMV Virus}

HEK293 cells were obtained from the Chinese Academy of Medical Sciences Cancer Institute and Hospital (CICAMS) after approximate 29 passages. These were grown in DMEM/high glucose medium (Hyclone) supplemented with 100 units $/ \mathrm{ml}$ penicillin $\mathrm{G}$ sodium, $100 \mu \mathrm{g} / \mathrm{ml}$ streptomycin, $4 \mathrm{mM} \mathrm{L}$-glutamine and $10 \%$ fetal bovine serum (Hyclone). PBMCs were obtained by $\mathrm{Fi}-$ coll-Hypaque gradient centrifugation (Pharmacia, Uppsala, Swe- den) from heparinized peripheral blood of healthy donors after informed consent. The HCMV experimental strain AD169 was provided by Cecilia Söderberg-Naucler, Karolinska Institute, Stockholm, Sweden, and was cultured on fibroblasts.

\section{Preparation of the Ad5F35-AD-1 Vaccine}

The design and construction of a recombinant adenovirus vaccine expressing the $\mathrm{AD}-1$ epitope of HCMV is shown in figure 1. Genomic DNA of the HCMV experimental strain AD169 was extracted using the Qiangen viral DNA extraction kit according to the manufacturer's instructions. The AD-1 gene was amplified from AD169 genomic DNA by polymerase chain reaction (PCR) with the following oligonucleotide primer pair: $5^{\prime}$-ATTG GGT ACC GCC ACC ATG ACC ATC AAC CAA ACC AGC GT3' and 5'-GCGA CTT AAG TCA ATG GTG ATG GTG ATG ATG CAT GCG TTT GAA GAG GTA G-3'; the forward primer incorporates a $K p n I$ site and a Kozak translation initiation sequence upstream of the start codon; the reverse primer introduces a hexahistidine tag and an AflII site. The AD-1 amplicon was ligated between the KpnI and AflII sites of shuttle plasmid pHMCMV5 (kindly provided by Hiroyuki Mizuguchi); the ligation mixture was transformed into $\mathrm{DH} 5 \alpha$ cells and selected by plating on LB agar/Kan plates (30 $\mathrm{gg} / \mathrm{ml}$ kanamycin). pHMCMV5-AD-1 was identified by restriction analysis and DNA sequencing.

The expression cassette, comprising the CMV promoter, AD1 and an SV40 polyA signal, was excised from the shuttle plasmid pHMCMV5-AD-1 with I-CeuI and PI-SceI and ligated into the E1/E3-deleted adenoviral backbone vector pAd5F35 (kindly provided by Hiroyuki Mizuguchi) cleaved with the same enzymes. The ligation mixture was treated with $S w a I$ to linearize non-recombinant pAd5F35 and selected on LB agar/Amp plates $(125 \mu \mathrm{g} /$ $\mathrm{ml}$ ampicillin). The presence of the expression cassette was verified by digestion with XhoI and by double-digestion with I-CeuI and PI-SceI. The resulting plasmids were also sequenced to confirm insertion of $\mathrm{AD}-1$.

pAd5F35-AD-1 was linearized with $\mathrm{PacI}$ and transfected into HEK293 cells using lipofectamine ${ }^{\text {TM }} 2000$ (Invitrogen) according to the manufacturer's instructions; pAd5F35-GFP was used as a control. Transfected HEK293 cells (HEK293-AD-1 or HEK293GFP) were incubated at $37^{\circ}$ for $7-10$ days until a maximal virus cytopathic effect was observed, and then were lysed by 3 freezethaw cycles. The cell lysate containing recombinant virus was added to $1.5 \times 10^{7}$ of preseeded confluent HEK293 cells at an MOI of 5. All subsequent infections in the amplification were performed in the same manner with identical MOIs until the 5th passage. The Ad5F35-AD-1 vaccine was purified by two rounds of $\mathrm{CsCl}$ density gradient ultracentrifugation. The titer of the large-scale Ad5F35-AD-1 virus preparation was established by plaque assay using HEK293 cells according to the protocol provided by Benyuanzhengyang Co. Ltd. (Beijing, PR China).

DNA was extracted from purified Ad5F35-AD-1 virions using a standard SDS-proteinase K lysis method. One microgram of the DNA sample was used to PCR amplify the AD-1 gene using primers described previously; PCR products were run on a $1.2 \%$ agarose gel.

AD-1 Expression in AD-1-Transfected HEK293 Cells

Total RNA from HEK293-AD-1 cells was isolated using the Trizol total RNA Isolation Reagent (Sangon, Shanghai, PR China) according to the manufacturer's instructions. One microgram of 
Fig. 1. Construction of the Ad5F35-AD-1 vaccine. The $\mathrm{gB} A \mathrm{AD}-1 \mathrm{DNA}$ sequence was amplified from the genome of HCMV AD169 by PCR, introducing a KpnI restriction site, a translation initiation (Kozak) sequence, a methionine start codon, the conserved epitope AD-1, a hexahistidine tag and an AflII restriction site. The amplicon was first cloned into shuttle plasmid pHMCMV5. The AD-1 expression cassette was then excised from pHMCMV5 and ligated into the pAd5F35 expression vector. The recombinant was packaged into infectious adenovirus by transfection of HEK293 cells, generating chimeric adenovirus Ad5F35-AD-1.

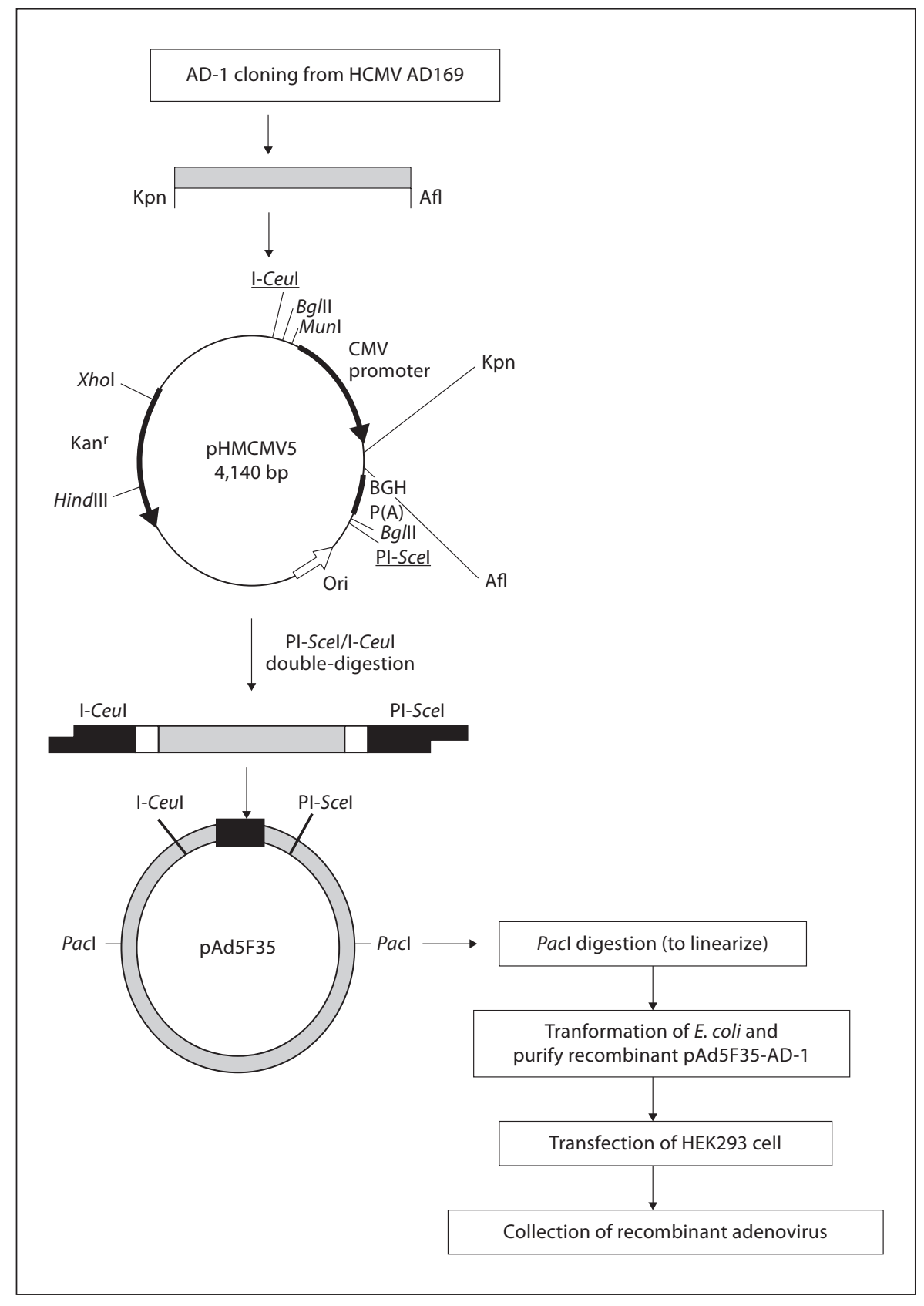

the RNA sample was used to prepare cDNA according to the protocol of the reverse transcription kit (Takara, Dalian, PR China). PCR was used to amplify the AD-1 gene and $\beta$-actin in a GeneAmp PCR system 2400 thermocycler (Perkin Elmer, Norwalk, Conn., USA). The amplified fragments were analyzed by electrophoresis on a $1.2 \%$ agarose gel.

To confirm AD-1 protein expression, Western blot analysis was conducted. HEK293-AD-1 cells were harvested 2 days after infection; HEK293-GFP and parental HEK293 cells served as negative controls. Cells were lysed, proteins collected using a RIPA Protein Extract Kit, and these were separated by 12\% SDS-
PAGE. After transfer to nitrocellulose the membranes were blocked (PBS containing 0.1\% Tween-20 and 5\% milk powder, $1 \mathrm{~h}$, room temperature) and incubated with primary rabbit polyclonal antibody to the $6 \times \mathrm{His}$ tag antibody (Abcam), 1:1,000, overnight at $4^{\circ}$. Membranes were then washed 3 times with PBST (PBS containing $0.1 \%(\mathrm{v} / \mathrm{v})$ Tween-20) and incubated with goat anti-rabbit IgG conjugated to horseradish peroxidase (GenScript Corporation) at 1:5,000 dilution in PBST for $1 \mathrm{~h}$ at room temperature. After washing extensively, the membranes were developed using an ECL Plus Western Blotting Detection System (GE Healthcare) and exposed to Kodak BioMax film. 


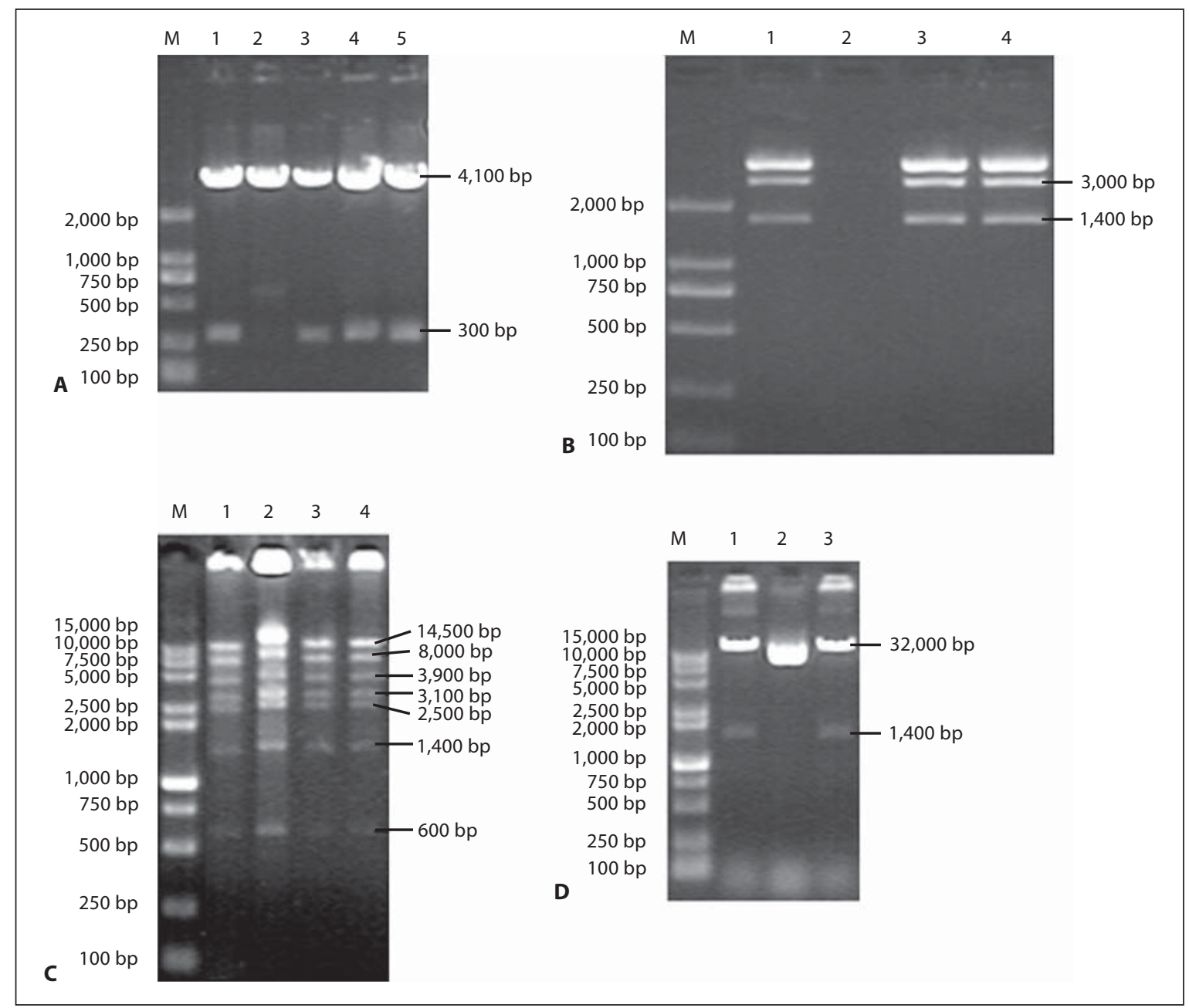

Fig. 2. Restriction endonuclease analysis of pHMCMV5-AD-1 and pAd5F35-AD-1. A pHMCMV5-AD-1 digested with KpnI/AflII: lanes 1, 3-5 are the recombinant shuttle plasmid pHMCMV5-AD-1 revealing the expected restriction fragments $(0.3+4.1 \mathrm{~kb})$. B pHMCMV5-AD-1 digested with I-CeuI/PI-SceI: lanes 1, 3, 4 are recombinant shuttle plasmids pHMCMV5-AD-1 showing restriction fragments of the expected sizes $(1.4+3.0 \mathrm{~kb})$. C XhoI restriction of recombinant pAd5F35-AD-1: lanes $1-4$ contain the expected 7 restriction fragments (14.5, 8.0, 3.9, 3.1, 2.5, 1.4 and 0.6 kb). D Plasmid pAd5F35-AD-1 digested with I-CeuI/PI-SceI: lanes 1 and 3 are recombinant pAd5F35-AD-1-generating restriction fragments of the expected sizes $(1.4+32 \mathrm{~kb})$.

In vitro Stimulation of PBMCs by Ad5F35-AD-1 Adenovirus Vaccine

PBMCs obtained from healthy blood donors were cultured in 6 -well plates $\left(2 \times 10^{6}\right.$ cells/well) in complete medium (RPMI 1640 supplemented with $10 \%$ fetal calf serum, FCS). Cells were infected with Ad5F35-GFP or Ad5F35-AD-1 at different MOIs (from $10^{2}$ to $10^{4}$ VP per cell) in RPMI 1640, $2 \%$ FCS. Six hours after infection, the cells were washed and resuspended into fresh complete medium. On days 1, 2, 6, and 10 after infection, transgene expression was analyzed by immunocytochemistry and cell viability was determined by trypan blue staining.
Expression of AD-1 in PBMCs Infected with Ad5F35-AD-1

Infected and non-infected PBMCs were placed on glass slides and fixed with $4 \%$ paraformaldehyde for $30 \mathrm{~min}$ at room temperature. Nonspecific antibody sites and Fc receptors were blocked with $10 \%$ normal goat serum for $30 \mathrm{~min}$. The cells were then incubated with the $6 \times$ His tag antibody, 1:1,000, overnight at $4^{\circ}$. Slides were washed 3 times with PBS and then incubated with goat anti-rabbit peroxidase-labelled secondary antibody for $10 \mathrm{~min}$ at $37^{\circ}$. All slides were processed by the SP method (Zhongshan Co. Ltd, Beijing, PR China) for $30 \mathrm{~min}$ at room temperature. Following staining with diaminobenzidone, slides were visualized using an Olympus-BX51 microscope. Nonspecific anti-IgG antibody was used as a negative control. 
Fig. 3. Cytopathogenic effect of adenovirus recombinants expressing either the $\mathrm{gB}$ AD-1 epitope of HCMV (A, B) or green fluorescent protein (C, D). Following transfection of HEK293 cells a normal cytopathogeniceffect was observed for HEK293AD-1 (A bright field; B dark field) and HEK293-GFP control cells (C bright field; D dark field); in D green fluorescence is observed. A-D $\times 400$.
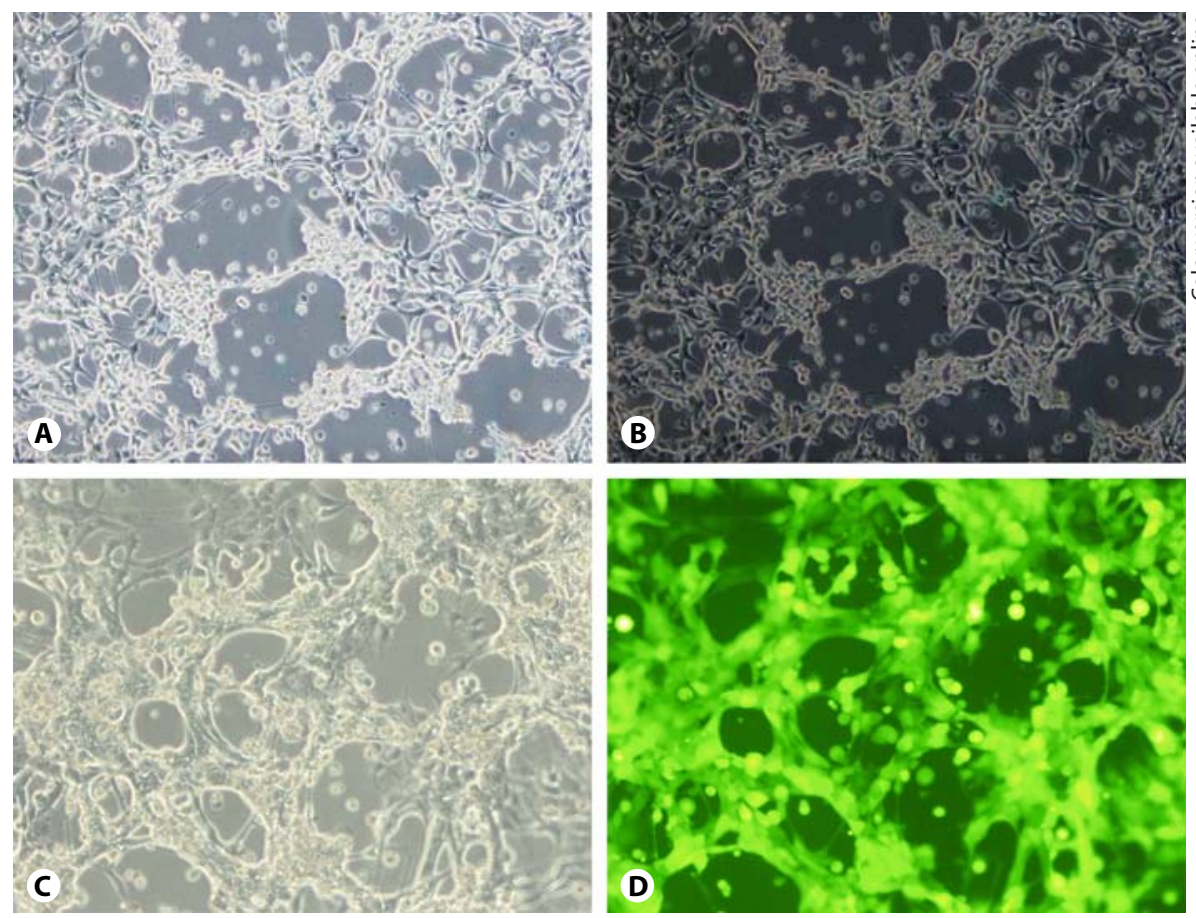

\section{Results}

Construction and Characterization of the

Recombinant Adenovirus Vector pAd5F35-AD-1

The AD-1-coding sequence was amplified from genomic DNA of HCMV strain AD169, sequenced, and cloned into the shuttle plasmid pHMCMV5. Double digestion of pHMCMV5-AD-1 with KpnI/AflI or I-CeuI/ PI-SceI produced fragments of the expected sizes $(0.3+$ 4.1 or $1.4+3.0 \mathrm{~kb}$, respectively), confirming the construction of pHMCMV5-AD-1 (fig. 2A, B).

The 1,140-bp AD-1 expression cassette from pHMCMV5-AD-1 was then cloned into the pAd5F35 expression vector by in vitro ligation to produce pAd5F35-AD-1. Digestion of pAd5F35-AD-1 with XhoI generated 7 fragments of the expected sizes $(14.5,8.0,3.9,3.1,2.5,1.4$ and $0.6 \mathrm{~kb}$; fig. 2C). Double digestion of pAd5F35-AD-1 with I-CeuI/PI-SceI also produced the two expected fragments (1.4 and $32 \mathrm{~kb}$; fig. 2D). Correct insertion of the AD-1 gene was further confirmed by DNA sequencing.

\section{Production of the Ad5F35-AD-1 Recombinant}

Adenovirus Vaccine

The recombinant pAd5F35-AD-1 vector and a control construct pAd5F35-GFP were both packaged in HEK293 cells; emerging recombinant adenoviruses Ad5F35-AD-1 and Ad5F35-GFP were cultivated. In both cases a normal cytopathic effect was observed. Efficient expression of green fluorescent protein (GFP) was observed 2 days after infection (fig. 3). Recombinant adenovirus was harvested from the transfected cells; the Ad5F35-AD-1 titer was 2.5 $\times 10^{9} \mathrm{PFU}$ as determined by plaque assay on HEK293 cells. PCR and gel electrophoresis revealed the anticipated AD-1 amplification product (302 bp) confirming the inclusion of $\mathrm{AD}-1$-coding sequences into recombinant adenovirus virions.

\section{Expression of AD-1 in HEK293 Cells Infected with Ad5F35-AD-1}

AD-1 expression in HEK293 cells infected with the recombinant virus was analyzed by RT-PCR. This generated the expected 302-bp fragment (fig. 4A). Protein expression was analyzed by Western blotting using antibody directed against the $6 \times \mathrm{His}$ tag. A band at $55 \mathrm{kDa}$, corresponding to the predicted size of the recombinant AD-1 protein $(55 \mathrm{kDa})$, was detected in extracts of cells (HEK293-AD-1) infected with Ad5F35-AD-1, but not in uninfected cells or in cells (HEK293-GFP) infected with the recombinant expressing GFP (fig. 4B). The monomer of $\mathrm{gB}$ consists of a surface component and a transmembrane component. AD-1 lies within the transmembrane component encompassing amino acids 552-635 (esti- 


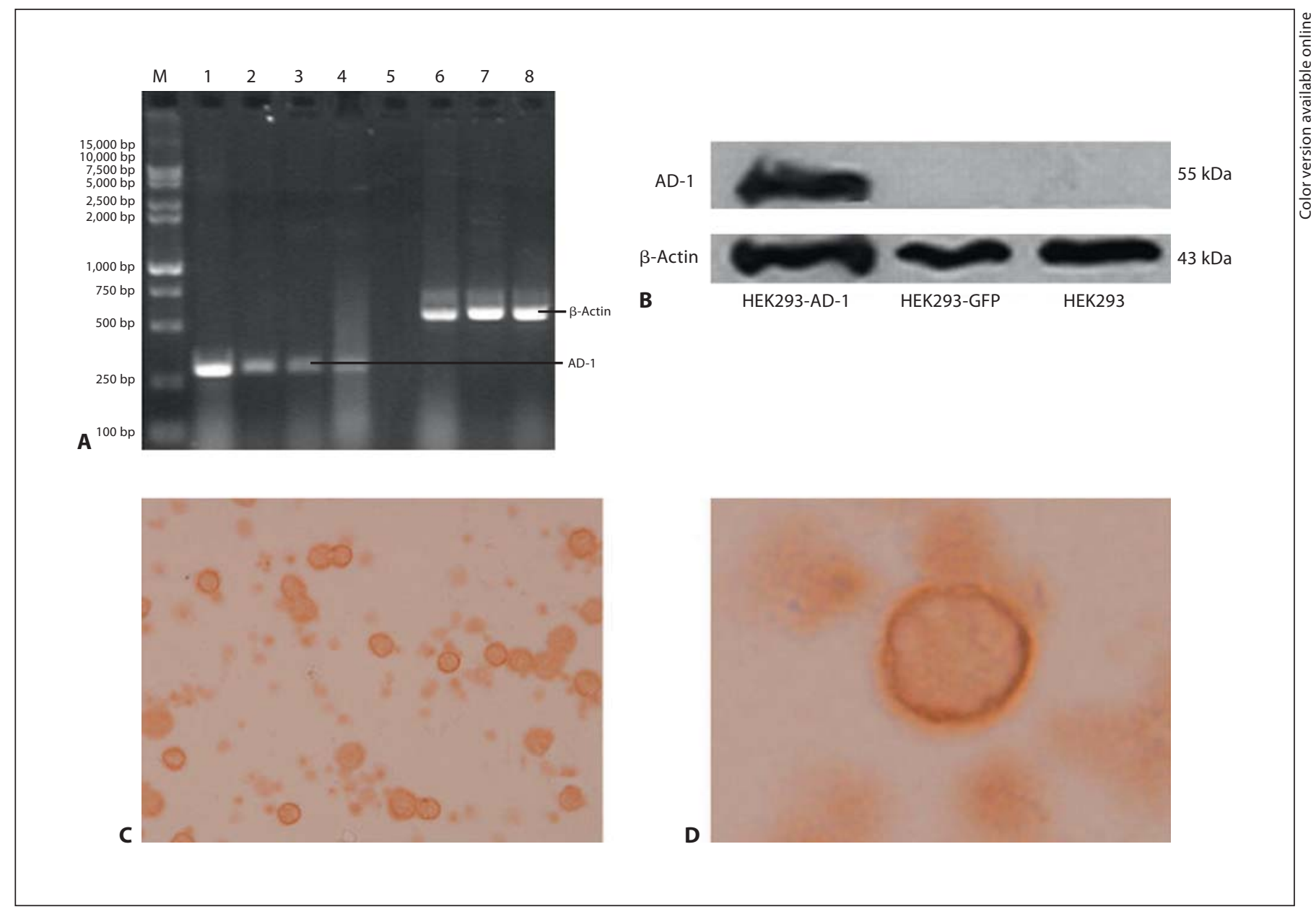

Fig. 4. Expression of the gB AD-1 epitope of HCMV from recombinant adenovirus Ad5F35-AD-1. A RT-PCR amplification of AD-1-coding sequences from infected HEK293 cells. Lanes 1-4 are the $\mathrm{AD}-1$ amplicon (302 bp); lanes $6-8$ the control $\beta$-actin amplicon $(578 \mathrm{bp})$. B Western blot of AD-1 protein expression. HEK293-AD-1 cells present the anticipated specific AD-1 (55
$\mathrm{kDa}$ ) protein band; no specific band was found in HEK293-GFP cells or parental HEK293 cells. C, D Immunocytochemistry of AD-1 expression in PBMCs. Strong expression of AD-1 antigen was recorded in PBMCs infected with Ad5F35-AD-1 (C). The typical peripheral (circular) pattern of staining demonstrates that recombinant AD-1 localizes to the cell membrane (D). mated molecular mass $30 \mathrm{kDa}$ ) [15]. The apparent size on gel electrophoresis, slightly larger than the predicted 30 $\mathrm{kD}$, may result from post-translational modification such as glycosylation which increases the size of the protein. This result indicates that deletion of the $\mathrm{gB}$ membranespanning domain and cytoplasmic tail does not alter the presentation of the AD-1 antigenic epitope.

\section{Transfection and Expression of AD-1 in PBMCs}

PBMCs were analyzed by immunocytochemistry using an antibody directed against the $6 \times$ His tag on days 1,2 , and 6 after infection. Strong expression of AD-1 antigen was recorded in cells infected with Ad5F35-AD-1; no expression was detected in control cells or in PBMCs infected with Ad5F35-GFP. The peripheral (circular) pattern of staining indicates that $\mathrm{AD}-1$ antigen localizes to the membrane of infected cells (fig. 4C, D). Expression of $\mathrm{AD}-1$ was time-dependent; the strongest expression was observed on day 6. Ad5F35-AD-1 had little detectable cytopathic activity on these cells. Viability was measured by trypan blue staining on days 1,6 , and 10 after infection; this failed to show any adverse effects of Ad5F35-AD-1 infection, with $90 \%$ of cells retaining viability. These results demonstrate that the $\mathrm{AD}-1$ epitope can be efficiently presented by PBMCs. 


\section{Discussion}

HCMV is a significant cause of human disease and methods to limit infection remain a high research priority. The present study describes a rational strategy for the construction of a recombinant HCMV vaccine based on the conserved AD-1 domain of glycoprotein $\mathrm{gB}$. We successfully generated a recombinant adenovirus, Ad5F35AD-1, that directs high-level expression of the conserved HCMV conformational epitope AD-1 under the control of the CMV promoter. We report that Ad5F35-AD-1 is stable and replicates to high titer in HEK293 cells. Ad5F35-AD-1 also efficiently infected human PBMCs and directed high-level expression of $\mathrm{AD}-1$ protein that localized to the membrane of infected cells.

In the development of a new HCMV vaccine it is important that the vaccine has high immunogenicity, low production cost, and low pathogenicity. Replication-defective Ad5F35 is one of the most promising vectors for HCMV vaccine development. The Ad5F35 vector contains a chimeric fiber gene encoding the fiber tail of Ad5 in combination with the fiber shaft and knob domains of Ad35. The hybrid virus replicates to titer in permissive tissue culture cells. Cell entry of Ad5F35 is thought to be mediated by $\mathrm{CD} 46$, a receptor expressed on most human cells [16]. Liver tropism and hepatoxicity are significantly lower for Ad5F35 than for Ad5 [17]. Importantly, Ad5F35 infection does not induce acute responses or the production of proinflammatory cytokines.

Ad5F35 has been used as a vaccine delivery vehicle in other infectious disease models including hepatitis C [18] and HIV [19], where potent transgene product-specific antibody and T-cell responses were reported. The vector is also being exploited in cancer immunotherapy $[20,21]$. In terms of efficacy, Ad5F35 appears to be more effective than other comparable vectors for recombinant protein expression in hematopoietic cells [12]. Our study has shown that Ad5F35-AD-1 efficiently infects freshly isolated $\mathrm{PBMCs}$ and directs high-level expression of the $\mathrm{AD}$ 1 epitope in these cells. The AD-1 polypeptide was correctly targeted to the cell membrane and expression increased over 6 days after infection. Cell viability was minimally affected by Ad5F35-AD-1 infection, and regardless of the multiplicity of infection. Furthermore, the Ad5F35-AD-1 recombinant elicited a potent lymphoproliferative response without affecting the viability of the PBMCs up to 10 days after infection.

An adenovirus-based system expressing a conformational epitope has a further advantage over traditional systems based on the expression of full-length antigens.
The truncated and secreted form of HCMV gB was reported to be more immunogenic in animals and humans than full-length $g B$, inducing significantly higher levels of virus-neutralizing antibodies than the full-length polypeptide $[7,8]$. Single epitope-based DNA vaccines can elicit specific immune responses against $\mathrm{T}$ - or B-cell epitopes in mice [22]. In addition, a vaccine based on a single epitope vaccine may avoid the problems of inaccurate cleavage and conformational presentation sometimes seen in vaccines encoding multiple epitopes. Expression of a single conformational epitope AD-1 may therefore improve immunogenicity. Experiments are underway to evaluate antigen-specific antibodies induction by Ad5F35-AD-1 in hu-SCID mice (data not shown).

In a clinical setting a severe problem is encountered in the case of women already seropositive for HCMV. During pregnancy such women are susceptible to re-infection with new HMCV strains, and this secondary infection can lead to symptomatic disease in the neonate [23]. Regarding vaccine development, it is possible that antibodies elicited by one vaccine type might fail to cross-neutralize different clinical isolates of HCMV. However, this may not be a major concern because sera from subjects vaccinated against gB/MF59 display broad cross-neutralization activity against diverse HCMV strains [7]. The AD-1 epitope is particularly well-conserved between different strains of HCMV. This may reflect the essential role it plays in gB folding and virus assembly. Indeed, AD-1 epitopes from different clinical isolates of HCMV exhibit little sequence variation [15]. Analysis of multiple clinical HCMV isolates with a panel of AD-1 monoclonal antibodies has revealed conservation of the $\mathrm{AD}-1$ antigenic epitope and antibody binding site in all tested HCMV isolates $[24,25]$. Expression of the conserved AD-1 epitope from Ad5F35 is therefore expected to elicit neutralizing immune responses against diverse strains of HCMV.

In conclusion, we have developed a recombinant vaccine against $\mathrm{HCMV}$ in which the conserved $\mathrm{AD}-1$ conformational epitope of HCMV gB protein is expressed from the adenovirus vector Ad5F35. The recombinant vaccine efficiently infects human PBMCs without affecting their viability. The vaccine may be a promising candidate for clinical trials and in prime-boost strategies in combination with other HCMV vaccine formulations.

\section{Acknowledgements}

This study was supported in part by grants from the National Natural Science Foundation (30600680) and the Shandong Technological Development Project (2005BS03022). 


\section{References}

-1 Staras SA, Flanders WD, Dollard SC, Pass RF, McGowan JE Jr, Cannon MJ: Seroprevalence of cytomegalovirus infection in the United States, 1988-1994. Clin Infect Dis 2006;43:1143-1151

$\checkmark 2$ Hebart H, Einsele H: Clinical aspects of CMV infection after stem cell transplantation. Hum Immunol 2004;65:432-436.

-3 Rowshani AT, Bemelman FJ, van Leeuwen EM, van Lier RA, ten Berge IJ: Clinical and immunologic aspects of cytomegalovirus infection in solid organ transplant recipients Transplantation 2005;79:381-386.

4 Limaye AP, Bakthavatsalam R, Kim HW, Kuhr CS, Halldorson JB, Healey PJ, Boeckh $\mathrm{M}$ : Late-onset cytomegalovirus disease in liver transplant recipients despite antiviral prophylaxis. Transplantation 2004;78:13901396.

5 Marshall GS, Rabalais GP, Stout GG, Waldeyer SL: Antibodies to recombinant-derived glycoprotein B after natural human cytomegalovirus infection correlate with neutralizing activity. J Infect Dis 1992;165:381384.

-6 Schleiss MR, Bourne N, Stroup G, Bravo FJ, Jensen NJ, Bernstein DI: Protection against congenital cytomegalovirus infection and disease in guinea pigs, conferred by a purified recombinant glycoprotein B vaccine. J Infect Dis 2004; 189:1374-1381.

7 Pass RF, Duliegè AM, Boppana S, Sekulovich R, Percell S, Britt W, Burke RL: A subunit cytomegalovirus vaccine based on recombinant envelope glycoprotein B and a new adjuvant. J Infect Dis 1999;180:970-975.

$>8$ Wang Z, La Rosa C, Maas R, Ly H, Brewer J, Mekhoubad S, Daftarian P, Longmate J, Britt WJ, Diamond DJ: Recombinant modified vaccinia virus Ankara expressing a soluble form of glycoprotein B causes durable immunity and neutralizing antibodies against multiple strains of human cytomegalovirus. J Virol 2004;78:3965-3976.
-9 Barrios Y, Knör S, Lantto J, Mach M, Ohlin $\mathrm{M}$ : Clonal repertoire diversification of a neutralizing cytomegalovirus glycoprotein Bspecific antibody results in variants with diverse anti-viral properties. Mol Immunol 2007;44:680-690.

10 Endrész V, Burián K, Berencsi K, Gyulai Z, Kari L, Horton H, Virok D, Méric C, Plotkin SA, Gönczöl E: Optimization of DNA immunization against human cytomegalovirus. Vaccine 2001;19:3972-3980.

-11 Mizuguchi H, Hayakawa T: Adenovirus vectors containing chimeric type 5 and type 35 fiber proteins exhibit altered and expanded tropism and increase the size limit of foreign genes. Gene 2002;285:69-77.

12 Yotnda P, Onishi H, Heslop HE, Shayakhmetov D, Lieber A, Brenner M, Davis A: Efficient infection of primitive hematopoietic stem cells by modified adenovirus. Gene Ther 2001;8:930-937.

13 Mizuguchi H, Hayakawa T: Targeted adenovirus vectors. Hum Gene Ther 2004;15: 1034-1044.

14 Gaggar A, Shayakhmetov DM, Lieber A: CD46 is a cellular receptor for group B adenoviruses. Nat Med 2003;9:1408-1412.

15 Britt WJ, Jarvis MA, Drummond DD, Mach $\mathrm{M}$ : Antigenic domain 1 is required for oligomerization of human cytomegalovirus glycoprotein B. J Virol 2005;79:4066-4079.

16 Gaggar A, Shayakhmetov DM, Lieber A: CD46 is a cellular receptor for group B adenoviruses. Nat Med 2003;9:1408-1412.

-17 Xin KQ, Jounai N, Someya K, Honma K, Mizuguchi H, Naganawa S, Kitamura K, Hayakawa T, Saha S, Takeshita F, Okuda K, Honda M, Klinman DM, Okuda K: Primeboost vaccination with plasmid DNA and a chimeric adenovirus type 5 vector with type 35 fiber induces protective immunity against HIV. Gene Ther 2005;12:1769-1777.
18 Thammanichanond D, Moneer S, Yotnda P, Aitken C, Earnest-Silveira L, Jackson D, Hellard M, McCluskey J, Torresi J, Bharadwaj M: Fiber-modified recombinant adenoviral constructs encoding hepatitis $\mathrm{C}$ virus proteins induce potent HCV-specific $\mathrm{T}$ cell response. Clinical Immunology 2008;128: 329-339.

19 Xin KQ, Sekimoto Y, Takahashi T, Mizuguchi H, Ichino M, Yoshida A, Okuda K: Chimeric adenovirus $5 / 35$ vector containing the clade C HIV gag gene induces a cross-reactive immune response against HIV. Vaccine 2007;25:3809-3815.

-20 Hoffmann D, Bayer W, Heim A, Potthoff A, Nettelbeck DM, Wildner O: Evaluation of twenty-one human adenovirus types and one infectivity-enhanced adenovirus for the treatment of malignant melanoma. J Invest Dermatol 2008;128:988-998.

21 Hoffmann D, Meyer B, Wildner O: Improved glioblastoma treatment with Ad5/35 fiber chimeric conditionally replicating adenoviruses. J Gene Med 2007;9:764-778.

$22 \mathrm{Xu} \mathrm{HB}, \mathrm{Xu}$ W, Chu YW, Wang Y, Xiong S: Single B or T-cell epitope-based DNA vaccine using modified vector induces specific immune response against hepadnavirus. Immunol Lett 2005;99:186-192.

23 Boppana SB, Rivera LB, Fowler KB, Mach M, Britt WJ: Intrauterine transmission of cytomegalovirus to infants of women with preconceptional immunity. N Engl J Med 2001; 344:1366-1371

24 Speckner A, Glykofrydes D, Ohlin M, Mach M: Antigenic domain 1 of human cytomegalovirus glycoprotein B induces a multitude of different antibodies which, when combined, results in incomplete virus neutralization. J Gen Virol 1999;80:2183-2191.

25 Ohlin M, Sundqvist VA, Mach M, Wahren B, Borrebaeck CA: Fine specificity of the human immune response to the major neutralization epitopes expressed on cytomegalovirus gp58/116 (gB), as determined with human monoclonal antibodies. J Virol 1993; 67:703-710 